Sharif University of Technology
Scientia Iranica
Transactions E: Industrial Engineering
SCIENTIA

Research Note

\title{
Monitoring of serially correlated processes using residual control charts
}

\author{
R. Osei-Aning, S.A. Abbasi* and M. Riaz \\ Department of Mathematics and Statistics, King Fahd University of Petroleum and Minerals, Dhahran, 31261, Saudi Arabia. \\ Received 5 September 2015; received in revised form 20 January 2016; accepted 20 June 2016
}

\author{
KEYWORDS \\ Autocorrelation; \\ Average run length; \\ CUSUM; \\ EWMA; \\ Extra quadratic loss; \\ Residuals.
}

\begin{abstract}
Control charts act as the most important tool for monitoring of process parameters. The assumption of independence that underpins the implementation of the charts is violated when process observations are correlated. The effect of this issue can lead to the malfunctioning of the usual control charts by causing a large number of false alarms or slowing the detection ability of the chart in unstable situations. In this paper, we investigated the performance of the Mixed EWMA-CUSUM and Mixed CUSUM-EWMA charts for the efficient monitoring of autocorrelated data. The charts are applied to the residuals obtained from fitting an autoregressive (AR) model to the autocorrelated observations. The performance of these charts is compared with the performances of the residual Shewhart, EWMA, CUSUM, combined Shewhart-CUSUM, and combined Shewhart-EWMA charts. Performance criteria such as Average Run Length (ARL) and Extra Quadratic Loss (EQL) are used for the evaluation and comparison of the charts. Illustrative examples are presented to demonstrate the application of the charts to serially correlated observations.

(C) 2017 Sharif University of Technology. All rights reserved.
\end{abstract}

\section{Introduction}

Control charts are typically used to observe irregularities that may occur in a production process so that corrective remedies are taken to curb the variation in order to produce products of good standards. Control charts usually have two limits that separate common from assignable causes of variation. Common causes in process monitoring are uncontrollable and random in nature; special causes, on the other hand, are components of the unnatural variations that are due to controllable factors such as operator error, worn out machine part, etc. Ever since Walter Andrew Shewhart [1] proposed the first control chart in the

\footnotetext{
*. Corresponding author.

E-mail addresses: roseianing@yahoo.com (R. Osei-Aning); saddamabbasi@yahoo.com (S.A.Abbasi);

riazm@kfupm.edu.sa (M.Riaz)
}

1920's for process monitoring, several charts have been developed over the years. The cumulative sum (CUSUM) and the Exponentially Weighted Moving Average (EWMA) charts developed by Page [2] and Roberts [3], respectively, are two of the most notable charts that are used in quality control to monitor small shifts in the process parameters.

The usual structures of control charts are based on the assumptions that the data produced from the process are independent and normally distributed [4]. The independence assumption is violated when observations from the process are serially correlated. This violation can lead to the malfunctioning of the control chart by causing a lot of false alarms or slowing the detection capability of the chart when a process is unstable $[5,6]$. Many processes in industrial and manufacturing operations produce observations which are serially correlated as a result of inertial elements and the frequent rate of sampling of the process $[4,5]$. 
Mason and Young [7] observed that most chemical processes in manufacturing operations were usually prone to producing highly correlated data, which was due to deterioration of machine part. Similarly, depletion of essential catalyst used in the process to speed up the industrial operations and contamination of equipment with chemicals produce correlated data [7]. A simple and less complicated way of controlling autocorrelation is less frequently sampling the process in order to break up the serial correlation phenomenon in the sequence of observations. The downside of this procedure is that it makes it hard to detect changes in the process since less information is available from the process. Therefore, in the presence of serial correlation, the use of residual charts, modified charts $[8,9]$, skip sampling strategy [10], etc. is the elaborate remedial technique that is adopted to deal with this phenomenon.

The concentration of this paper is on the use of residual charts. In this approach, an appropriate time series model is fitted to the correlated data. The conventional charts are then applied to the residuals of the model, which are expected to be uncorrelated. This procedure makes it possible to transform existing charts into residual control charts. The concept of using time series models in control charts was advanced by Alwan and Roberts [11]. Other works such as $[5,6,8,12-21]$ and the references therein considered serially correlated observations that could be modelled with autoregressive integrated moving average models (ARIMA) using residual control charts. A benefit of the residual chart is that it can be utilized in any form of serially correlated observations, be it stationary or otherwise $[5,16]$. However, a disadvantage of the residual chart is that knowledge of time series modelling is needed in order to fit an appropriate model to the correlated observations. In Statistical Process Control (SPC) applications, difficulties associated with residual charts such as choosing an appropriate model and estimating the parameters of the model make the use of this charting technique challenging.

Over the years, great emphasis has been put on the studies of the Shewhart, CUSUM, and EWMA charts for location monitoring in the presence of serial correlation. Harris and Ross [6] examined the effect of correlation on the performance of the EWMA and CUSUM charts. They noted that errors might be generated if the correlation behavior of the data was not accounted for in the charts. Alwan and Roberts [11] developed the Shewhart residual chart for monitoring of the correlated data. Wardel et al. [14] considered serial correlation that could be fitted with ARMA (1, 1) by comparing the performance of the Shewhart and EWMA charts with that of the common-cause and special-cause control charts. Karaoglan and Bayhan [16] focused their work on autocorrelation that could be fitted with a trend AR (1) and AR (1) process models using Shewhart, CUSUM, and EWMA residual charts. Following their previous work, they studied the performance of these charts for trend AR (1) model using vegetable oil data from industry [17]. They noted that for small to moderate shifts, the EWMA and CUSUM residual charts performed better than the Shewhart type charts for a moderate and strong positively correlated process. Lu and Reynolds $[19,20]$ investigated the performance of the CUSUM and EWMA residual charts in the respective works using an AR (1) model plus a random error term. Lin [22] considered autocorrelation that could be modelled with $\operatorname{AR}(1)$ and IMA $(1,1)$ models by applying the forecast errors from these models to the Shewhart, CUSUM, EWMA, combined Shewhart-CUSUM (CSCUSUM), and combined Shewhart-EWMA (CSEWMA) charts. The combined charts retained the positive strengths of the Shewhart and EWMA or CUSUM charts with respect to small and large process shifts. Zhang [23] proposed the EWMA chart for stationary process (EWMAST) and compared the performance of this chart with that of the modified and residual Shewhart charts for $\operatorname{AR}(1), \operatorname{ARMA}(1,1)$, and $\operatorname{AR}(2)$ process models. Subsequently, Zhang [5] compared the EWMAST and modified Shewhart charts with the Shewhart, CUSUM, and EWMA residual charts for an AR (1) process model. He noted that the EWMAST chart performed relatively better for small to medium mean shifts when the process was autocorrelated.

Recently, Abbas et al. [24] and Zaman et al. [25] proposed the Mixed EWMA-CUSUM (MEC) and Mixed CUSUM-EWMA (MCE) charts, respectively, for monitoring processes for which the observations were independent and normally distributed. However, these charts malfunction in the presence of serial correlation by producing frequent false alarms. In this paper, we present the MEC and MCE residual charts for monitoring observations that can be modelled with an autoregressive process. Comparisons among the charts are conducted for different autocorrelation and mean shift levels using individual observations.

The rest of the paper is structured as follows; Section 2 describes the structures of various residual charts for monitoring an autocorrelated process; the performance evaluation and comparison of the charts are briefly presented in Section 3; illustrative examples to demonstrate how the charts operate are presented in Section 4; and, finally, conclusions are summarized in Section 5.

\section{Residual charts for AR (1) processes}

Serial correlation describes the relationship that exists between a variable and its past values at different time 
lags. In the residual chart, an appropriate time series model is fitted to the data. Subsequently, the control charts are applied to the residuals $\left(e_{t}\right)$ of the model which are expected to be randomly distributed. We will restrict this work to control chart structures for serially correlated observations that can be modelled with an autoregressive AR (1) model. Several works on serial correlation in control charts have used this model (e.g. [5,18,26]). The general AR (1) model is represented as:

$$
X_{t}-\mu=\phi_{1}\left(X_{t-1}-\mu\right)+\varepsilon_{t},
$$

where $X_{t}$ is the observed time series at time $(t), \varepsilon_{t}$ is the white noise term, $\mu$ is the mean, and $\phi_{1}$ is the autocorrelation coefficient. $\varepsilon_{t}$ is assumed to be independently and normally distributed with mean 0 and variance $\sigma_{\varepsilon}^{2}$ (i.e. $\varepsilon_{t} \sim N\left(0, \sigma_{\varepsilon}^{2}\right)$ ). To allow for the process mean shift, we include a time varying mean to Eq. (1) following [26]. This yields:

$$
X_{t}=\mu_{t}+\phi_{1}\left(X_{t-1}-\mu_{t-1}\right)+\varepsilon_{t} .
$$

Theoretically, in order to model assignable causes in the charts, a mean shift of magnitude $(\delta)$ is put into Eq. (2) such that $\mu$ shifts to $\mu+\delta$. The resulting sequence of residuals [26] is obtained by:

$$
\begin{aligned}
& e_{t}=X_{t}-\widehat{X}_{t}, \\
& \begin{cases}e_{t}=\varepsilon_{t}+\delta & t=1 \\
e_{t}=\varepsilon_{t}+\left(1-\phi_{1}\right) \delta & t>1\end{cases}
\end{aligned}
$$

where $\widehat{X_{t}}=\mu\left(1-\phi_{1}\right)+\phi_{1} X_{t-1}$ with the assumption that the coefficient estimates are absolutely accurate. $\widehat{X}_{t}$ is the predicted value of $X_{t}$. Therefore, $e_{t}$ are used instead of the original observations $\left(X_{t}\right)$ in the control charts.

\subsection{Control charts for residuals}

In this section, control chart structures for the Shewhart, CUSUM, EWMA, CSCUSUM, CSEWMA, MEC, and MCE residual charts are briefly discussed for the correlated process.

\subsubsection{Shewhart residual control chart}

The Shewhart chart [1] is efficient for monitoring large shifts in the process mean. In the presence of serial correlation, the Shewhart residual chart $[11,18]$ is used to handle the correlation. In this chart, $e_{t}$ in Eq. (3b) is used as the plotting statistic. A process is said to be out-of-control in the chart when the plotting statistic $\left(e_{t}\right)$ falls outside the following control limits:

$$
\mathrm{UCL}=\mu+L_{s} \sigma_{e} \quad \text { and } \quad \mathrm{LCL}=\mu-L_{s} \sigma_{e},
$$

where $L_{s}$ is a control chart multiplier, which is used to adjust the in-control run length of the chart. $\mu$ and $\sigma_{e}$ represent the in-control mean and standard deviation of the residuals from an AR (1) process, respectively.

\subsubsection{CUSUM residual control chart}

The CUSUM chart [2] is best noted for detecting small changes in the mean of the process. However, in the presence of serial correlation, this chart is transformed into the CUSUM residual chart to account for the correlation which affects the process $[6,18,19]$. The two sided CUSUM statistics in the residual chart are defined as:

$$
\left\{\begin{array}{l}
C_{t}^{+}=\max \left(0, e_{t}-\mu-K+C_{t-1}^{+}\right) \\
C_{t}^{-}=\max \left(0, \mu-e_{t}-K+C_{t-1}^{-}\right)
\end{array}\right.
$$

We define $K=k \sigma_{e}$ and $H=h \sigma_{e}$, where $K$ and $H$ denote the reference and the decision interval values, respectively. $k=0.5$ is a widely used figure because it makes the CUSUM chart very sensitive to small and medium shifts [4]. The chart is considered to be in an out-of-control state when either of the CUSUM statistics exceeds $H$, i.e. when:

$$
C_{t}^{+}>H \quad \text { or } \quad C_{t}^{-}>H
$$

\subsubsection{EWMA residual control chart}

The EWMA chart [3] was developed for monitoring small shifts in the process. When observations from the process are serially correlated, the EWMA residual chart is used $[6,18,20]$. For the EWMA residual chart, the statistic $\left(W_{t}\right)$ is defined as:

$$
W_{t}=\lambda e_{t}+(1-\lambda) W_{t-1},
$$

where $\lambda$ is the smoothing parameter $(0<\lambda \leq 1)$. Small values of $\lambda$ are desirable for the detection of small shifts and vice versa. The variance of $W_{t}$ statistic is given as:

$$
\begin{aligned}
\operatorname{Var}\left(W_{t}\right) & =\sigma_{W_{t}}^{2}=\lim _{t \rightarrow \infty} \sigma_{e}^{2}\left(\frac{\lambda}{2-\lambda}\right)\left[1-(1-\lambda)^{2 t}\right] \\
& =\sigma_{e}^{2}\left(\frac{\lambda}{2-\lambda}\right)
\end{aligned}
$$

An out-of-control signal is generated if $W_{t}$ falls outside the following control limits:

$$
\begin{aligned}
& \mathrm{UCL}=\mu+L_{E} \sigma_{e} \sqrt{\left(\frac{\lambda}{2-\lambda}\right)} \text { and } \\
& \mathrm{LCL}=\mu-L_{E} \sigma_{e} \sqrt{\left(\frac{\lambda}{2-\lambda}\right)}
\end{aligned}
$$

where $L_{E}$ is the control constant in the chart. 


\subsubsection{CSCUSUM residual control chart}

The CSCUSUM chart [27] combines the schemes of the CUSUM and Shewhart control charts. Therefore, it is very sensitive for monitoring small and large mean shifts. In the presence of serial correlation, the CSCUSUM residual chart was proposed by Lin [22]. A process is said to be out-of-control when the current observation $\left(e_{t}\right)$ falls outside the Shewhart limits or any of the CUSUM statistics exceeds the value of $H$, i.e. when:

$$
\left\{\begin{array}{l}
e_{t}>\mathrm{UCL}=\mu+L_{s} \sigma_{e} \quad \text { or } \\
e_{t}<\mathrm{LCL}=\mu-L_{s} \sigma_{e} \quad \text { or } \\
C_{t}^{+}>H \text { or } C_{t}^{-}>H
\end{array}\right.
$$

\subsubsection{CSEWMA residual control chart}

The CSEWMA chart [28] operates by combining the Shewhart and EWMA schemes. This combined chart is also very sensitive to small and large shifts in the process mean. The CSEWMA residual chart is used for location monitoring when the observations are correlated [22]. This residual chart is considered outof-control when the EWMA statistic $\left(W_{t}\right)$ falls outside the EWMA limits or the current observation $\left(e_{t}\right)$ falls outside the Shewhart limits, i.e. when:

$$
\left\{\begin{array}{l}
e_{t}>\mathrm{UCL}=\mu+L_{s} \sigma_{e} \quad \text { or } \\
e_{t}<\mathrm{LCL}=\mu-L_{s} \sigma_{e} \quad \text { or } \\
W_{t}>\mathrm{UCL}=\mu+L_{E} \sigma_{e} \sqrt{\left(\frac{\lambda}{2-\lambda}\right)} \text { or } \\
W_{t}<\mathrm{LCL}=\mu-L_{E} \sigma_{e} \sqrt{\left(\frac{\lambda}{2-\lambda}\right)}
\end{array}\right.
$$

\subsubsection{MEC residual control chart}

The MEC chart [24] integrates the EWMA statistic $\left(W_{t}\right)$ into the CUSUM statistic. This chart is effective for detecting small shifts in the process. In this study, we present the $\mathrm{MEC}$ residual chart for monitoring serially correlated observations. This residual chart is represented as:

$$
\left\{\begin{array}{l}
\operatorname{MEC}_{t}^{+}=\max \left(0, W_{t}-\mu-K_{t}+\mathrm{MEC}_{t-1}^{+}\right) \\
\operatorname{MEC}_{t}^{-}=\max \left(0, \mu-W_{t}-K_{t}+\mathrm{MEC}_{t-1}^{-}\right)
\end{array}\right.
$$

We define $K_{t}=k^{*} \sigma_{W}$ and $H_{t}=h^{*} \sigma_{W}$, where $K_{t}$ and $H_{t}$ denote the reference and decision interval values, respectively. This chart is said to be out-of-control when either of the MEC statistics exceeds the value of $H_{t}$, i.e. when:

$$
\mathrm{MCE}_{t}^{+}>H_{t} \quad \text { or } \quad \mathrm{MEC}_{t}^{-}>H_{t}
$$

\subsubsection{MCE residual control chart}

The MCE chart [25] is formulated by incorporating the CUSUM statistics into the EWMA statistic. It retains the good characteristics of both CUSUM and EWMA charts and eliminates the inability of both charts to detect large shifts when used in isolation. This chart is noted to perform relatively better than the Shewhart chart for large shifts. To eliminate the usual problems associated with control chart utilization in the presence of serial correlation, this MCE chart is transformed into the MCE residual chart to improve process performance. The proposed transformed chart is defined as:

$$
\left\{\begin{array}{l}
\mathrm{MCE}_{t}^{+}=\lambda C_{t}^{+}+(1-\lambda) \mathrm{MCE}_{t-1}^{+} \\
\mathrm{MCE}_{t}^{-}=\lambda C_{t}^{-}+(1-\lambda) \mathrm{MCE}_{t-1}^{-}
\end{array}\right.
$$

where $C_{t}^{+}$and $C_{t}^{-}$represent the CUSUM statistics given in Eq. (5). The starting values of the MCE statistics in Eq. (14) are set equal to the grand mean of $C_{t}^{+}$and $C_{t}^{-}$, respectively. That is:

$$
\mathrm{MCE}_{0}^{+}=\mathrm{MCE}_{0}^{-}=\mu_{c} .
$$

The mean and variance of the MCE statistics are time varying and are obtained through a simulated procedure. They are represented as:

$$
\begin{aligned}
& \operatorname{mean}\left(\mathrm{MCE}_{t}^{+}\right)=\operatorname{mean}\left(\mathrm{MCE}_{t}^{-}\right)=\mu_{\mathrm{MCE}}, \\
& \operatorname{Var}\left(\mathrm{MCE}_{t}^{+}\right)=\operatorname{Var}\left(\mathrm{MCE}_{t}^{-}\right)=\sigma_{\mathrm{MCE}_{t}}^{2}
\end{aligned}
$$

However, as $t \rightarrow \infty$, the target mean of the CUSUM statistics becomes equal to the target mean of the MCE statistics. That is, $\mu_{\mathrm{MCE}}=\mu_{C}$. An out-ofcontrol signal is generated if either $\mathrm{MCE}_{t}^{+}$or $\mathrm{MCE}_{t}^{-}$ falls outside the control limit, i.e. when:

$$
\begin{aligned}
& \mathrm{MCE}_{t}^{+}>\mathrm{UCL}_{t}=\mu_{\mathrm{MCE}_{t}}+L_{M} \sigma_{\mathrm{MCE}_{t}} \text { or } \\
& \mathrm{MCE}_{t}^{-}>\mathrm{UCL}_{t}=\mu_{\mathrm{MCE}_{t}}+L_{M} \sigma_{\mathrm{MCE}_{t}},
\end{aligned}
$$

where $L_{M}$ is the control constant in the chart.

\section{Performance evaluation and comparison of the charts}

The performance evaluation and comparison of all the residual charts discussed in Section 2 will be done using the Average Run Length (ARL) and Extra Quadratic Loss (EQL) performance criteria. ARL is the mean number of samples that must be plotted before a point signals an out-of-control condition [5]. For an incontrol process, ARL is denoted by $\mathrm{ARL}_{0}$ and this quantifies the false alarm rate. An out-of-control state is represented as $\mathrm{ARL}_{1}$ and a chart with the smallest 
$\mathrm{ARL}_{1}$ value is considered very sensitive to process shifts in comparison with its counterparts at a fixed $\mathrm{ARL}_{0}$ value [29]. EQL is the weighted mean ARL over a range of the process shifts $\left(\delta_{\min }<\delta<\delta_{\max }\right)$ using the square of the shift $\left(\delta^{2}\right)$ as the weight [30]. The EQL is represented numerically as:

$$
\mathrm{EQL}=\frac{1}{\delta_{\max }-\delta_{\min }} \int_{\delta_{\min }}^{\delta_{\max }} \delta^{2} \operatorname{ARL}(\delta) d \delta .
$$

The approximate value of EQL will be determined numerically by using the Trapezium rule. The Trapezium rule is a method that is used to calculate the area under a curve by dividing it into series of strips with equal width. Therefore, the estimated value represents the approximate value of the integral. A control chart with the smallest EQL value has better performance ability than its counterparts over an entire shift range. The ARL and EQL performance measures [29,30] are regularly used in process evaluation and comparison of different control charts. To determine these performance criteria, we consider $\delta=0,0.5,1,1.5,2,2.5,3,3.5,4$ as the values of the mean shift and:

$$
\phi=0, \pm 0.25, \pm 0.50, \pm 0.75, \pm 0.90,
$$

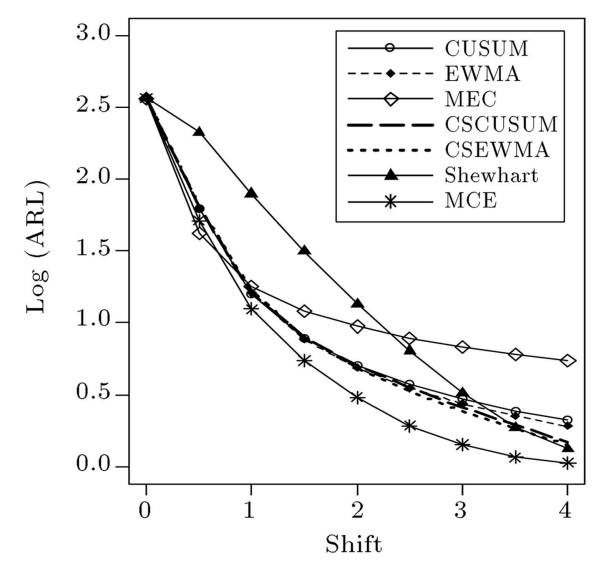

(a) $\phi=0.25$

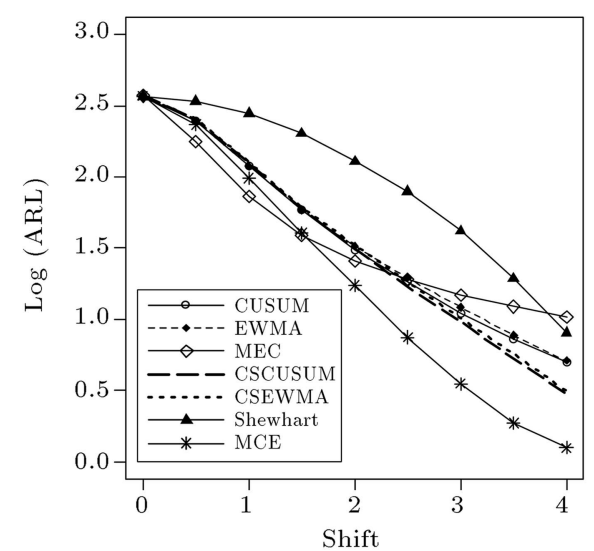

(c) $\phi=0.75$ asthe values of the autocorrelation coefficient. We categorize the mean shift by small $(\delta<1)$, medium $(1 \leq \delta<3)$, and large $(\delta \geq 3)$.

For each level of mean shift and autocorrelation coefficient considered, 10000 simulations are done to obtain the ARL values. All the design parameters in the charts are adjusted in order to have in-control ARL of 370 . We use $\left(L_{s}=3\right),(k=0.5, h=4.77),\left(L_{E}=\right.$ $2.86, \lambda=0.2),\left(L_{s}=3.5, k=0.5, h=4.914\right),\left(L_{s}=\right.$ $\left.3.5, L_{E}=2.91, \lambda=0.2\right),(k=0.5, h=21.28, \lambda=0.2)$, and $\left(k=0.5, \lambda=0.2, L_{M}=4.18\right)$ design parameters in the Shewhart, CUSUM, EWMA, CSCUSUM, CSEWMA, MEC, and MCE charts, respectively. The specific value of $L_{s}=3.5$ is used in the combined charts because Lucas and Saccucci $[27,28]$ recommend a value greater than the Shewhart limit of 3 (i.e., 3.5 or 4) since a larger value has a smaller effect on the incontrol ARL. The ARL and EQL values for the residual charts are displayed in Tables 1 and 2 for an AR (1) process with $\phi \geq 0$ and $\phi<0$, respectively. In this work, we have replicated the ARL results of the EWMA and CUSUM charts from the work of [5] to ensure the validity of the simulation procedures used. Figures 1 and 2 present graphical comparison of the charts for different autocorrelation and mean shift levels.

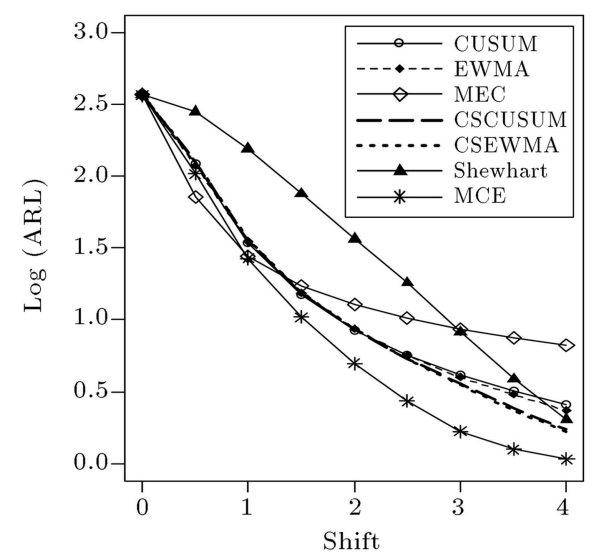

(b) $\phi=0.50$

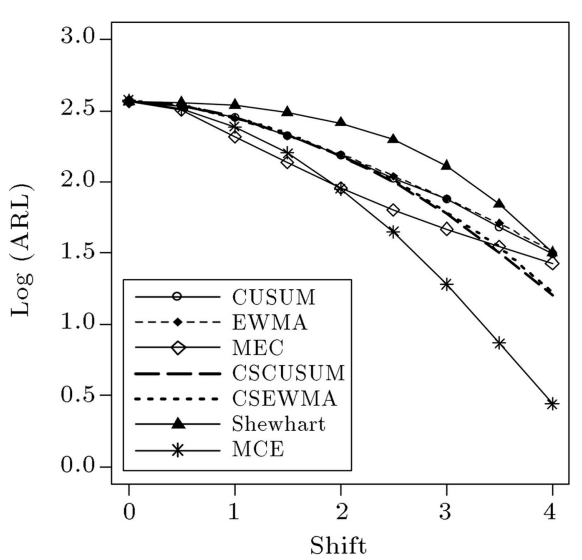

(d) $\phi=0.90$

Figure 1. ARL curves for different residual charts for positively correlated processes. 
Table 1. ARLs and EQLs for different residual charts at different levels of $\delta$ when $\mathrm{ARL}_{0}=370$ and $\phi \geq 0$.

\begin{tabular}{|c|c|c|c|c|c|c|c|c|}
\hline$\phi$ & $\delta$ & CSCUSUM & CSEWMA & MEC & CUSUM & EWMA & Shewhart & MCE \\
\hline \multirow{10}{*}{0} & 0 & 372.21 & 374.95 & 374.32 & 370.95 & 371.47 & 370.43 & 369.12 \\
\hline & 0.5 & 36.53 & 37.64 & 29.17 & 34.90 & 35.71 & 155.59 & 29.37 \\
\hline & 1 & 10.13 & 10.14 & 13.92 & 9.96 & 9.86 & 43.64 & 7.77 \\
\hline & 1.5 & 5.54 & 5.30 & 9.76 & 5.49 & 5.23 & 15.10 & 3.82 \\
\hline & 2 & 3.77 & 3.53 & 7.81 & 3.84 & 3.57 & 6.40 & 2.35 \\
\hline & 2.5 & 2.79 & 2.62 & 6.61 & 3.00 & 2.78 & 3.20 & 1.66 \\
\hline & 3 & 2.15 & 2.03 & 5.79 & 2.48 & 2.31 & 2.00 & 1.32 \\
\hline & 3.5 & 1.69 & 1.63 & 5.20 & 2.15 & 2.02 & 1.46 & 1.14 \\
\hline & 4 & 1.35 & 1.33 & 4.74 & 1.96 & 1.81 & 1.20 & 1.06 \\
\hline & EQL & 14.38 & 13.86 & 33.70 & 16.19 & 15.28 & 25.95 & 9.73 \\
\hline \multirow{10}{*}{0.25} & 0 & 371.64 & 372.25 & 367.61 & 368.92 & 371.29 & 368.76 & 367.36 \\
\hline & 0.5 & 63.69 & 65.16 & 41.84 & 62.02 & 62.66 & 212.95 & 51.40 \\
\hline & 1 & 16.17 & 16.88 & 17.96 & 15.88 & 16.25 & 79.67 & 12.51 \\
\hline & 1.5 & 8.00 & 7.91 & 12.10 & 7.86 & 7.72 & 31.46 & 5.46 \\
\hline & 2 & 5.06 & 4.83 & 9.42 & 5.09 & 4.88 & 13.56 & 3.02 \\
\hline & 2.5 & 3.54 & 3.33 & 7.81 & 3.76 & 3.51 & 6.41 & 1.92 \\
\hline & 3 & 2.56 & 2.42 & 6.77 & 2.95 & 2.73 & 3.25 & 1.42 \\
\hline & 3.5 & 1.94 & 1.82 & 6.01 & 2.44 & 2.26 & 1.86 & 1.16 \\
\hline & 4 & 1.47 & 1.43 & 5.43 & 2.10 & 1.92 & 1.33 & 1.06 \\
\hline & EQL & 18.86 & 18.32 & 40.02 & 20.78 & 19.81 & 45.09 & 12.14 \\
\hline \multirow{10}{*}{0.5} & 0 & 370.64 & 370.03 & 371.50 & 370.57 & 373.04 & 373.14 & 369.30 \\
\hline & 0.5 & 124.86 & 126.04 & 72.30 & 122.09 & 118.65 & 281.82 & 104.28 \\
\hline & 1 & 35.74 & 36.55 & 27.92 & 34.39 & 35.26 & 155.52 & 26.92 \\
\hline & 1.5 & 15.21 & 15.70 & 17.27 & 14.96 & 15.37 & 75.76 & 10.46 \\
\hline & 2 & 8.58 & 8.62 & 12.74 & 8.51 & 8.69 & 36.62 & 4.96 \\
\hline & 2.5 & 5.40 & 5.31 & 10.23 & 5.68 & 5.64 & 18.26 & 2.71 \\
\hline & 3 & 3.60 & 3.50 & 8.67 & 4.15 & 4.00 & 8.23 & 1.67 \\
\hline & 3.5 & 2.43 & 2.35 & 7.54 & 3.18 & 3.02 & 3.89 & 1.25 \\
\hline & 4 & 1.74 & 1.67 & 6.70 & 2.55 & 2.34 & 2.03 & 1.07 \\
\hline & EQL & 30.67 & 30.58 & 52.96 & 33.10 & 32.65 & 99.38 & 19.02 \\
\hline \multirow{10}{*}{0.75} & 0 & 372.81 & 367.22 & 371.71 & 369.14 & 374.74 & 368.99 & 369.62 \\
\hline & 0.5 & 254.53 & 256.65 & 176.23 & 248.91 & 246.11 & 340.48 & 233.68 \\
\hline & 1 & 124.03 & 127.94 & 72.39 & 120.40 & 118.97 & 278.85 & 98.04 \\
\hline & 1.5 & 60.05 & 61.16 & 39.14 & 58.70 & 58.76 & 202.28 & 40.40 \\
\hline & 2 & 31.23 & 33.20 & 25.84 & 30.71 & 32.61 & 128.75 & 17.28 \\
\hline & 2.5 & 16.99 & 18.45 & 18.95 & 18.35 & 19.52 & 79.20 & 7.47 \\
\hline & 3 & 9.49 & 10.14 & 14.87 & 10.95 & 12.14 & 41.60 & 3.51 \\
\hline & 3.5 & 5.35 & 5.69 & 12.26 & 7.21 & 7.72 & 19.32 & 1.86 \\
\hline & 4 & 2.99 & 3.14 & 10.37 & 5.00 & 5.14 & 8.04 & 1.25 \\
\hline & EQL & 91.10 & 95.49 & 99.16 & 97.39 & 101.25 & 313.06 & 53.44 \\
\hline \multirow{10}{*}{0.9} & 0 & 367.95 & 372.95 & 368.22 & 368.32 & 370.32 & 368.61 & 374.28 \\
\hline & 0.5 & 348.31 & 349.73 & 321.05 & 341.38 & 338.49 & 361.48 & 328.62 \\
\hline & 1 & 282.32 & 284.35 & 208.82 & 284.59 & 281.10 & 346.13 & 244.32 \\
\hline & 1.5 & 214.37 & 219.47 & 137.76 & 210.75 & 209.98 & 309.44 & 159.49 \\
\hline & 2 & 151.76 & 151.34 & 91.01 & 153.31 & 156.03 & 259.59 & 89.19 \\
\hline & 2.5 & 99.73 & 103.94 & 63.93 & 106.71 & 110.09 & 198.20 & 44.72 \\
\hline & 3 & 59.61 & 60.54 & 46.76 & 75.20 & 76.47 & 129.35 & 19.09 \\
\hline & 3.5 & 32.09 & 34.93 & 35.31 & 48.20 & 51.32 & 69.58 & 7.35 \\
\hline & 4 & 15.90 & 16.76 & 26.92 & 31.33 & 32.20 & 31.84 & 2.75 \\
\hline & EQL & 392.37 & 403.42 & 303.93 & 455.28 & 465.61 & 710.13 & 200.68 \\
\hline
\end{tabular}


Table 2. ARLs and EQLs for different residual charts at different levels of $\delta$ when $\mathrm{ARL}_{0}=370$ and $\phi<0$.

\begin{tabular}{|c|c|c|c|c|c|c|c|c|}
\hline$\phi$ & $\delta$ & CSCUSUM & CSEWMA & MEC & CUSUM & EWMA & Shewhart & MCE \\
\hline \multirow{10}{*}{-0.25} & 0 & 373.31 & 367.77 & 370.16 & 372.57 & 372.84 & 372.30 & 367.55 \\
\hline & 0.5 & 23.23 & 24.80 & 22.61 & 22.86 & 23.68 & 113.18 & 19.59 \\
\hline & 1 & 7.43 & 7.19 & 11.61 & 7.26 & 7.03 & 25.59 & 5.64 \\
\hline & 1.5 & 4.39 & 4.10 & 8.43 & 4.41 & 4.10 & 8.20 & 3.04 \\
\hline & 2 & 3.12 & 2.91 & 6.85 & 3.24 & 2.99 & 3.75 & 2.05 \\
\hline & 2.5 & 2.40 & 2.27 & 5.86 & 2.62 & 2.43 & 2.26 & 1.55 \\
\hline & 3 & 1.90 & 1.83 & 5.19 & 2.24 & 2.10 & 1.66 & 1.28 \\
\hline & 3.5 & 1.55 & 1.53 & 4.67 & 2.03 & 1.93 & 1.33 & 1.14 \\
\hline & 4 & 1.32 & 1.31 & 4.23 & 1.91 & 1.78 & 1.16 & 1.05 \\
\hline & EQL & 12.15 & 11.77 & 29.76 & 14.07 & 13.26 & 17.75 & 8.65 \\
\hline \multirow{10}{*}{-0.5} & 0 & 369.21 & 369.00 & 371.40 & 367.71 & 373.22 & 373.03 & 367.08 \\
\hline & 0.5 & 16.81 & 17.62 & 18.59 & 16.42 & 16.79 & 81.54 & 13.98 \\
\hline & 1 & 5.95 & 5.68 & 10.11 & 5.88 & 5.57 & 15.52 & 4.51 \\
\hline & 1.5 & 3.70 & 3.43 & 7.52 & 3.74 & 3.48 & 5.11 & 2.65 \\
\hline & 2 & 2.71 & 2.56 & 6.17 & 2.87 & 2.64 & 2.68 & 1.90 \\
\hline & 2.5 & 2.13 & 2.06 & 5.33 & 2.38 & 2.22 & 1.89 & 1.50 \\
\hline & 3 & 1.77 & 1.74 & 4.76 & 2.09 & 2.01 & 1.53 & 1.27 \\
\hline & 3.5 & 1.51 & 1.51 & 4.24 & 1.98 & 1.91 & 1.31 & 1.14 \\
\hline & 4 & 1.31 & 1.31 & 3.99 & 1.90 & 1.78 & 1.16 & 1.06 \\
\hline & EQL & 10.94 & 10.70 & 27.06 & 12.88 & 12.21 & 13.63 & 8.10 \\
\hline \multirow{10}{*}{-0.75} & 0 & 369.81 & 369.20 & 370.10 & 369.45 & 371.61 & 371.21 & 369.53 \\
\hline & 0.5 & 13.04 & 13.25 & 16.24 & 12.74 & 12.70 & 59.70 & 10.72 \\
\hline & 1 & 5.04 & 4.73 & 9.11 & 5.02 & 4.68 & 10.19 & 3.87 \\
\hline & 1.5 & 3.21 & 3.03 & 6.87 & 3.34 & 3.08 & 3.60 & 2.38 \\
\hline & 2 & 2.41 & 2.30 & 5.69 & 2.62 & 2.41 & 2.21 & 1.80 \\
\hline & 2.5 & 1.97 & 1.92 & 4.95 & 2.21 & 2.10 & 1.76 & 1.48 \\
\hline & 3 & 1.71 & 1.70 & 4.38 & 2.03 & 1.97 & 1.51 & 1.27 \\
\hline & 3.5 & 1.51 & 1.50 & 4.02 & 1.96 & 1.90 & 1.31 & 1.13 \\
\hline & 4 & 1.32 & 1.31 & 3.83 & 1.90 & 1.78 & 1.16 & 1.05 \\
\hline & EQL & 10.24 & 10.03 & 25.19 & 12.19 & 11.60 & 11.50 & 7.76 \\
\hline \multirow{10}{*}{-0.9} & 0 & 370.84 & 369.50 & 370.02 & 372.47 & 372.14 & 368.76 & 370.62 \\
\hline & 0.5 & 11.30 & 11.53 & 15.00 & 11.16 & 11.17 & 50.01 & 9.43 \\
\hline & 1 & 4.57 & 4.34 & 8.62 & 4.60 & 4.32 & 8.17 & 3.55 \\
\hline & 1.5 & 3.00 & 2.84 & 6.54 & 3.16 & 2.91 & 3.11 & 2.29 \\
\hline & 2 & 2.28 & 2.20 & 5.44 & 2.51 & 2.32 & 2.06 & 1.75 \\
\hline & 2.5 & 1.91 & 1.89 & 4.76 & 2.15 & 2.05 & 1.73 & 1.47 \\
\hline & 3 & 1.69 & 1.69 & 4.19 & 2.00 & 1.97 & 1.51 & 1.27 \\
\hline & 3.5 & 1.50 & 1.50 & 3.96 & 1.96 & 1.90 & 1.31 & 1.14 \\
\hline & 4 & 1.31 & 1.31 & 3.66 & 1.90 & 1.78 & 1.16 & 1.05 \\
\hline & EQL & 9.92 & 9.78 & 24.25 & 11.90 & 11.37 & 10.70 & 7.63 \\
\hline
\end{tabular}

\subsection{ARL criterion}

\subsubsection{Positively correlated observations}

For positively correlated processes $(\phi>0)$, the MEC residual chart, followed by the MCE residual charts, outperforms the other charts for small shifts in the process mean. Please note that the CUSUM, EWMA, CSCUSUM, and CSEWMA residual charts perform the same and almost quite well for small shifts. The Shewhart residual chart is the worst performing chart for small shifts.
Again, the MCE performs better than the other charts with respect to medium shifts $(1 \leq \delta<3)$. It is worth pointing out that when $\phi=0.75$ and $\phi=0.90$, the MEC residual chart also performs creditably well for medium shifts, especially between $1 \leq \delta<2$. The performances of the CUSUM, EWMA, CSCUSUM, and CSEWMA residual charts are almost the same for medium shifts but inferior to the MCE residual chart.

With large shifts in the mean of positively correlated processes, the MCE performs exceptionally well 


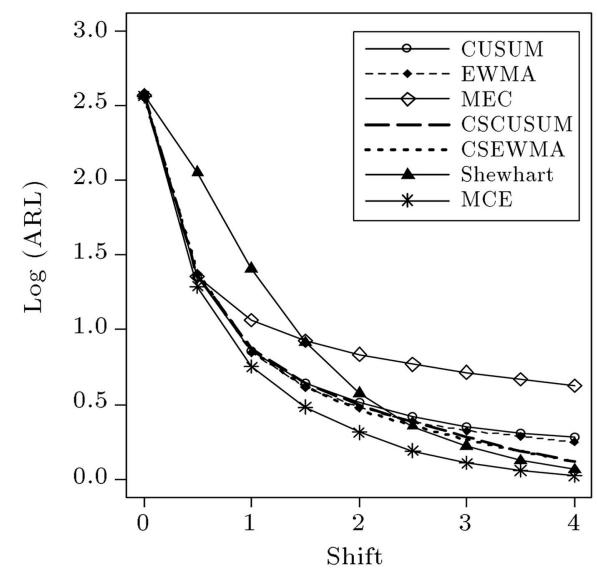

(a) $\phi=-0.25$

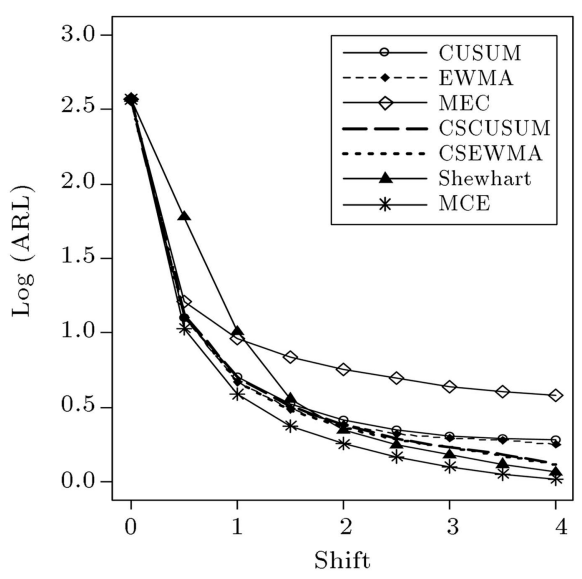

(c) $\phi=-0.75$

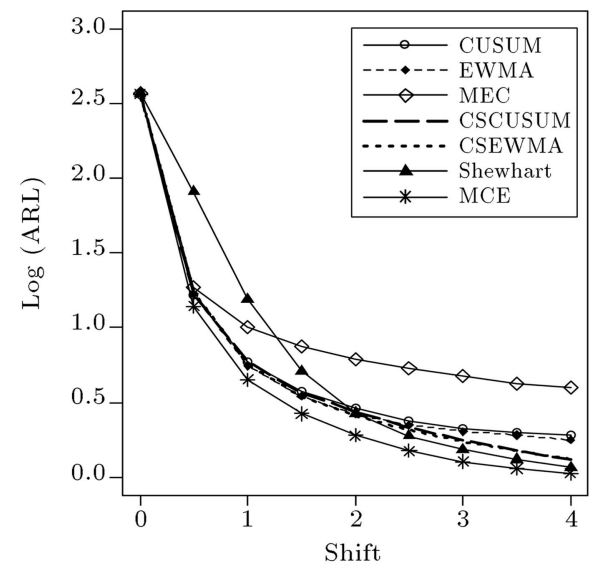

(b) $\phi=-0.50$

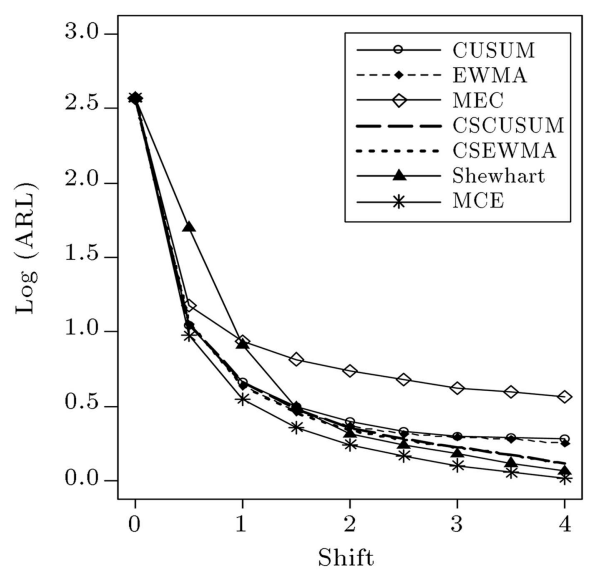

(d) $\phi=-0.90$

Figure 2. ARL curves for different residual charts for negatively correlated processes.

and better than the other charts. Generally, the CSCUSUM and CSEWMA residual charts also perform better than the EWMA, CUSUM, and Shewhart residual charts. For highly correlated processes (i.e., $\phi \geq 0.75)$, the performance of the Shewhart residual chart is the worst for large shifts.

\subsubsection{Negatively correlated observations.}

When a process is negatively autocorrelated $(\phi<$ 0 ), all the charts (MCE, MEC, EWMA, CUSUM, CSCUSUM, and CSEWMA) except the Shewhart residual chart perform creditably well for small shifts in the process. However, the MEC residual chart mostly performs well for $\delta \leq 0.5$.

Furthermore, the MCE residual chart performs better than the other charts for medium shifts in the process mean. The performances of the EWMA, CUSUM, CSCUSUM, and CSEWMA residual charts are almost the same for medium shifts but inferior to the MCE residual chart. The Shewhart residual chart does perform quite well like both CSCUSUM and CSEWMA residual charts with $2<\delta<3$. The MEC chart is the worst performing chart for this shift category.
For large shifts, the MCE and MEC residual charts are the best and worst residual charts for negatively correlated processes, respectively. However, the Shewhart, CSCUSUM, and CSEWMA residual charts perform equally well for large shifts as expected.

\section{2. $E Q L$ criterion}

Lastly, with in-control ARL fixed at 370 in all the competing charts, it is observed that the MCE residual chart, followed by the CSCUSUM and CSEWMA residual charts, consistently has the smallest EQL values for both positive and negative autocorrelated processes. This indicates that the MCE residual chart has the best performance ability over the shift range with respect to the correlated process. It is clear that for positively and negatively correlated processes, the Shewhart and MEC residual charts are respectively the worst performing charts.

\section{Illustrative examples}

In this section, we consider both the simulated and reallife data sets for the illustration of the control charts when a sustained shift is introduced in the process 
mean of the AR (1) observations. In the illustrations, we use the initial-state ARL as the performance measure for the comparison of the charts.

Example 1: Simulated data. We simulated 100 observations of an AR (1) model with $\phi=0.5$. The residuals of this model were standardized. At observation 83, a persistent shift of magnitude $1 \sigma_{e}$ was introduced in the observations. The first 82 observations represent the in-control state of the process. Based on these residuals, we constructed different charts as described in Section 2. Various parameters in the residual charts were chosen such that the incontrol ARL $=370$. The graphical displays of the charts are presented in Figure 3. We have omitted the graphical displays for the CSCUSUM and CSEWMA residual charts, because their outputs are similar to the outputs of the CUSUM and EWMA residual charts,

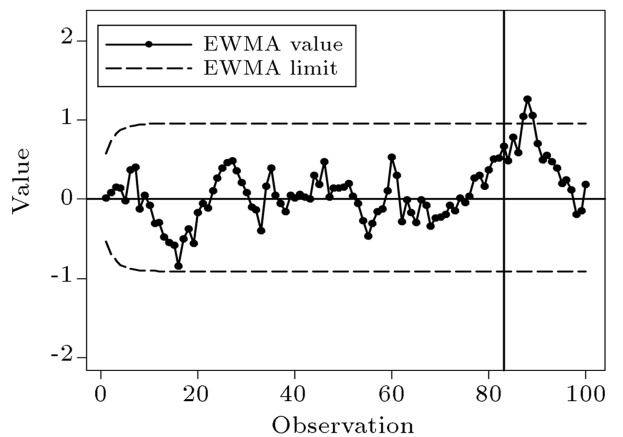

(a) EWMA

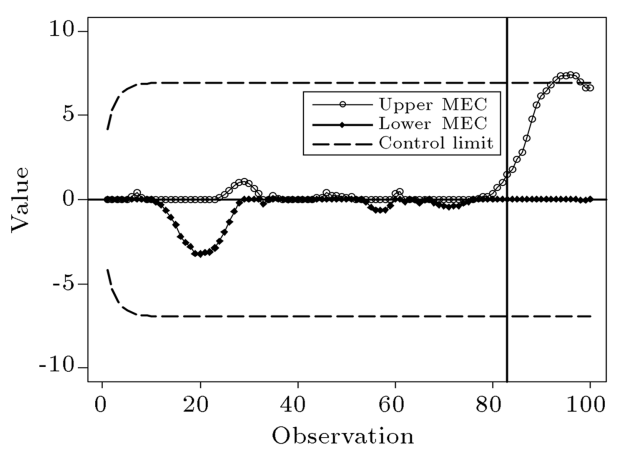

(c) MEC
Table 3. Out-of-control points for the different residual charts based on the data in Example 1.

\begin{tabular}{ll}
\hline Charts & Out-of-control points \\
\hline Shewhart & 0 \\
EWMA & $87,88,89$ \\
CUSUM & 88,89 \\
MEC & $93,94,95,96,97,98$ \\
MCE & $89,90,91,92,93,94,95$ \\
\hline
\end{tabular}

respectively. Table 3 displays various charts with their corresponding out-of-control points. Due to the limits of space, we have provided 35 of the 100 values of each charting statistic computed in Table 4.

It is clear from Table 3 that after the $82 \mathrm{nd}$ observation, the MCE, MEC, EWMA, CUSUM, and Shewhart residual charts detected $7,6,3,2$, and 0 outof-control points, respectively. The MCE and MEC

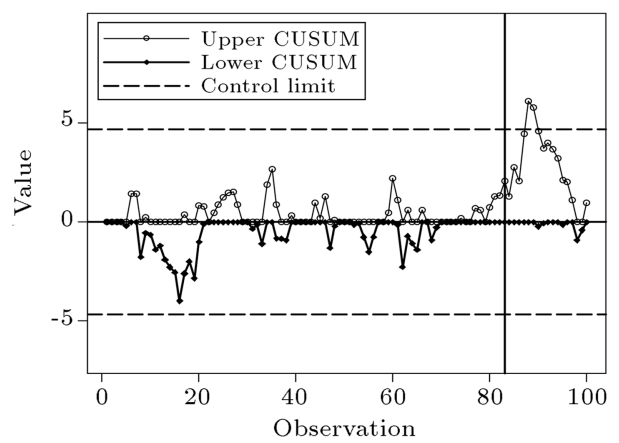

(b) CUSUM

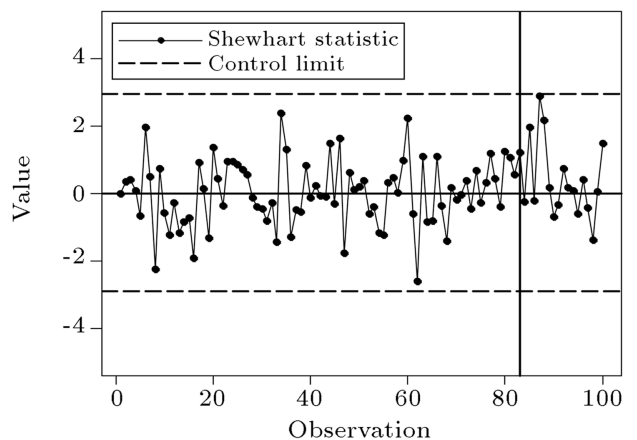

(d) Shewhart

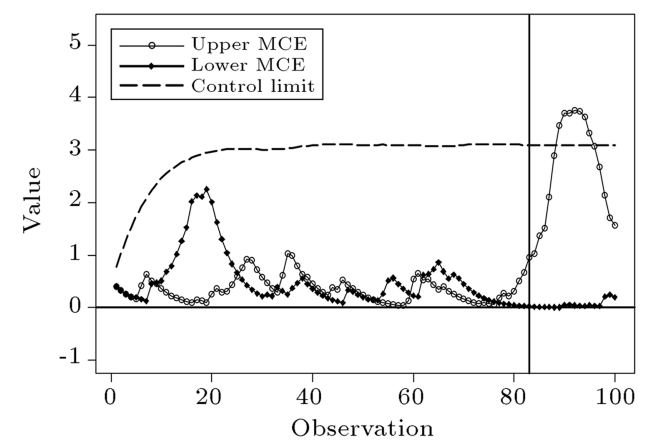

(e) $\mathrm{MCE}$

Figure 3. Residual charts for simulated data in Example 1. 
Table 4. The values of the charting statistics (35 out of 100 observations).

\begin{tabular}{|c|c|c|c|c|c|c|c|c|}
\hline \multirow{2}{*}{ Observation } & \multirow{2}{*}{$\begin{array}{c}\text { Shewhart } \\
e_{t}\end{array}$} & \multirow{2}{*}{$\begin{array}{c}\text { EWMA } \\
W_{t}\end{array}$} & \multicolumn{2}{|c|}{ CUSUM } & \multicolumn{2}{|c|}{ MEC } & \multicolumn{2}{|c|}{ MCE } \\
\hline & & & $C_{t}^{+}$ & $C_{t}^{-}$ & $\mathrm{MEC}_{t}^{+}$ & $\mathrm{MEC}_{t}^{-}$ & $\mathrm{MCE}_{t}^{+}$ & $\mathrm{MCE}_{t}^{-}$ \\
\hline 1 & 0.00 & 0.02 & 0.00 & 0.00 & 0.00 & 0.00 & 0.40 & 0.40 \\
\hline 2 & 0.36 & 0.09 & 0.00 & 0.00 & 0.00 & 0.00 & 0.32 & 0.32 \\
\hline 3 & 0.41 & 0.15 & 0.00 & 0.00 & 0.00 & 0.00 & 0.26 & 0.26 \\
\hline 4 & 0.09 & 0.14 & 0.00 & 0.00 & 0.00 & 0.00 & 0.21 & 0.21 \\
\hline 5 & -0.66 & -0.02 & 0.00 & 0.19 & 0.00 & 0.00 & 0.17 & 0.20 \\
\hline 6 & 1.96 & 0.38 & 1.45 & 0.00 & 0.19 & 0.00 & 0.42 & 0.16 \\
\hline 7 & 0.51 & 0.40 & 1.45 & 0.00 & 0.42 & 0.00 & 0.63 & 0.13 \\
\hline 8 & -2.24 & -0.12 & 0.00 & 1.77 & 0.11 & 0.00 & 0.50 & 0.46 \\
\hline 9 & 0.75 & 0.05 & 0.24 & 0.55 & 0.00 & 0.00 & 0.45 & 0.48 \\
\hline 10 & -0.56 & -0.07 & 0.00 & 0.64 & 0.00 & 0.00 & 0.36 & 0.51 \\
\hline 11 & -1.22 & -0.30 & 0.00 & 1.40 & 0.00 & 0.16 & 0.29 & 0.69 \\
\hline 12 & -0.27 & -0.30 & 0.00 & 1.21 & 0.00 & 0.32 & 0.23 & 0.79 \\
\hline 13 & -1.18 & -0.47 & 0.00 & 1.91 & 0.00 & 0.65 & 0.18 & 1.02 \\
\hline 14 & -0.84 & -0.55 & 0.00 & 2.29 & 0.00 & 1.06 & 0.15 & 1.27 \\
\hline 15 & -0.73 & -0.58 & 0.00 & 2.55 & 0.00 & 1.50 & 0.12 & 1.53 \\
\hline 16 & -1.91 & -0.85 & 0.00 & 3.99 & 0.00 & 2.20 & 0.09 & 2.02 \\
\hline 17 & 0.91 & -0.50 & 0.40 & 2.61 & 0.00 & 2.56 & 0.16 & 2.14 \\
\hline 18 & 0.14 & -0.37 & 0.02 & 2.01 & 0.00 & 2.79 & 0.13 & 2.11 \\
\hline 19 & -1.30 & -0.56 & 0.00 & 2.84 & 0.00 & 3.21 & 0.10 & 2.26 \\
\hline 20 & 1.37 & -0.17 & 0.86 & 1.01 & 0.00 & 3.24 & 0.25 & 2.01 \\
\hline 21 & 0.44 & -0.05 & 0.78 & 0.10 & 0.00 & 3.14 & 0.36 & 1.63 \\
\hline 22 & -0.37 & -0.11 & 0.00 & 0.00 & 0.00 & 3.12 & 0.29 & 1.30 \\
\hline 23 & 0.96 & 0.10 & 0.45 & 0.00 & 0.00 & 2.87 & 0.32 & 1.04 \\
\hline 24 & 0.94 & 0.27 & 0.88 & 0.00 & 0.08 & 2.46 & 0.43 & 0.83 \\
\hline 25 & 0.88 & 0.39 & 1.24 & 0.00 & 0.29 & 1.93 & 0.59 & 0.67 \\
\hline 26 & 0.73 & 0.46 & 1.46 & 0.00 & 0.56 & 1.33 & 0.77 & 0.53 \\
\hline 27 & 0.58 & 0.48 & 1.52 & 0.00 & 0.86 & 0.71 & 0.92 & 0.43 \\
\hline 28 & -0.11 & 0.36 & 0.90 & 0.00 & 1.03 & 0.21 & 0.91 & 0.34 \\
\hline 29 & -0.38 & 0.22 & 0.00 & 0.00 & 1.06 & 0.00 & 0.73 & 0.27 \\
\hline 30 & -0.46 & 0.08 & 0.00 & 0.00 & 0.96 & 0.00 & 0.58 & 0.22 \\
\hline 31 & -0.81 & -0.10 & 0.00 & 0.34 & 0.67 & 0.00 & 0.47 & 0.24 \\
\hline 32 & -0.26 & -0.13 & 0.00 & 0.14 & 0.36 & 0.00 & 0.37 & 0.22 \\
\hline 33 & -1.44 & -0.39 & 0.00 & 1.11 & 0.00 & 0.25 & 0.30 & 0.40 \\
\hline 34 & 2.39 & 0.16 & 1.88 & 0.00 & 0.00 & 0.00 & 0.62 & 0.32 \\
\hline 35 & 1.31 & 0.39 & 2.67 & 0.00 & 0.21 & 0.00 & 1.03 & 0.26 \\
\hline
\end{tabular}

residual charts were slow in detecting the initial two out-of-control signals as compared to the CUSUM and EWMA residual charts; but, in all, they detected the most signals. According to this example, the MCE residual chart was the best performing chart, followed by the MEC and the EWMA residual charts. The observations in this example are consistent with the findings in Section 4.

Example 2: Real-life data. In this example, we use a real-life dataset to demonstrate how the charts perform. In the production of a certain equipment, several tests of insulation resistance are carried out on a material which was selected for use. In an Insulation Resistance (IR) test, Direct Current (DC) voltage is passed through the dielectric material. An IR tester, which is connected to the material, records the IR measurements. The data [1] consists of 204 observations of resistance (in megohms) taken on samples of the material. An AR (1) process model with 


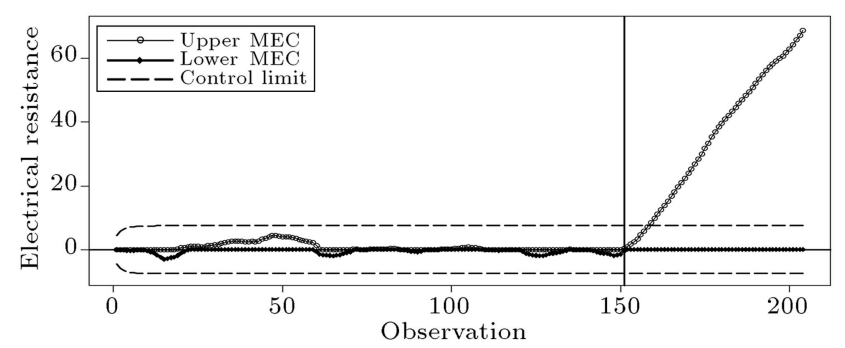

(a) MEC

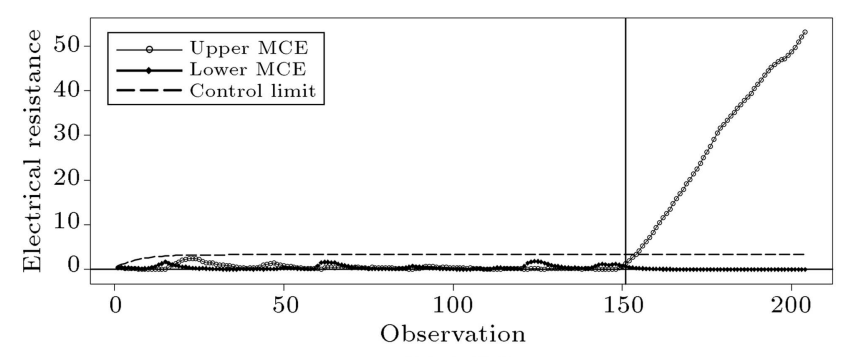

(b) MEC

Figure 4. Residual charts for real-life data in Example 2.

$\phi=0.55$ provided a perfect fit to the data. The residuals obtained from the model were standardized. At observation 151, a step shift of $2.5 \sigma_{e}$ was introduced into the observations. Figure 4 displays the outputs of the MEC and MCE residual charts, respectively. After the 150th observation, the MCE and MEC charts signalled 51 and 46 out-of-control points, respectively. This shows the superiority of the MCE residual chart to the MEC residual chart with respect to medium shifts. The findings in this example are also consistent with the results in Section 4.

\section{Conclusions}

Many situations arise in manufacturing and industrial operations when the observations become correlated. In this paper, we investigated and compared the performances of the MEC and MCE residual charts with those of the existing Shewhart, CUSUM, EWMA, CSCUSUM, and CSEWMA residual charts. The comparisons indicated that for the AR (1) process, the MEC residual chart is good for detecting small mean shifts of positively correlated processes. Further, the MEC residual chart is not sensitive to the detection of medium and large shifts as compared to other charts. Also, the comparisons revealed that the MCE residual chart performed better for small and medium shifts than the CUSUM, EWMA, and the combined residual charts did whilst, for large mean shifts, the MCE residual chart performed better than the Shewhart and the other residual charts did for the AR (1) process. We emphasize that the performances of the residual charts depend on the process model, correlation levels, and the magnitude of the shift.

These charts can be applied in processes involved in the chemical industry, smelting, and refinery operations, which are known to produce correlated data for monitor quality characteristics. Future research can concentrate on the optimization designs of the MEC and MCE charts for correlated processes and the application of theses charts to serially correlated multivariate data can be studied. Further, the steadystate run length behavior of these charts can also be investigated.

\section{References}

1. Shewhart, W.A. Economic Control of Quality Manufactured Product, Van Nostrand, New York (1931).

2. Page, E.S. "Continuous inspection schemes", Biometrika, 41(1-2), pp. 100-115 (1954).

3. Roberts, S.W. "Control chart tests based on geometric moving averages", Technometrics, 1(3), pp. 239-250 (1959).

4. Montgomery, D.C., Introduction to Statistical Quality Control, 6th Ed., John Willey \& Sons, New York (2009).

5. Zhang, N.F. "Statistical control charts for monitoring the mean of a stationary process", Journal of Statistical Computation and Simulation, 66(3), pp. 249-258 (2000).

6. Harris, T.J. and Ross, W.H. "Statistical process control procedures for correlated observations", The Canadian Journal of Chemical Engineering, 69(1), pp. 48-57 (1991).

7. Mason, R.L. and Young, J.C., Multivariate Statistical Process Control with Industrial Applications, Society for Industrial and Applied Mathematics, Philadelphia, PA (2001).

8. Kramer, H. and Schmid, W. "Control charts for time series", Nonlinear Analysis, Theory, Methods and Applications, 30(7), pp. 4007-4016 (1997).

9. Vasilopoulos, A.V. and Stamboulis, A.P. "Modification of control chart limits in the presence of data correlation", Journal of Quality Technology, 10(1), pp. 20-30 (1978).

10. Costa, A.F.B. and Castagliola, P. "Effect of measurement error and autocorrelation on the X chart", Journal of Applied Statistics, 38(4), pp. 661-673 (2011).

11. Alwan, L.C. and Roberts, H.V. "Time-series process modeling for statistical control", Journal of Business and Economic Statistics, 6(1), pp. 87-95 (1988).

12. Montgomery, D.C. and Mastrangelo, C.M. "Some statistical process control methods for autocorrelated data", Journal of Quality Technology, 23(3), pp. 179193 (1991).

13. English, J.R., Lee, S.C., Martin, T.W. and Tilmon, C. "Detecting changes in autoregressive processes with Xbar and EWMA charts", IIE Transactions, 32(12), pp. 1103-1113 (2000). 
14. Wardell, D.G., Moskowitz, H. and Plante, R.D. "Control charts in the presence of data correlation", Management Science, 38(8), pp. 1084-1105 (1992).

15. Koehler, A.B., Marks, N.B. and O'Connell, R.T. "EWMA control charts for autoregressive processes", Journal of the Operational Research Society, 52(6), pp. 699-707 (2001).

16. Karaoglan, A.D. and Bayhan, G.M. "Performance comparison of residual control charts for trend stationary first order autoregressive processes", Gazi University Journal of Science, 24(2), pp. 329-339 (2011).

17. Karaoglan, A.D. and Bayhan, G.M. "ARL performance of residual control charts for trend AR (1) process: A case study on peroxide values of stored vegetable oil", Scientific Research and Essays, 7(13), pp. 1405-1414 (2012).

18. Wieringa, J.E. "Statistical process control for serially correlated data", Ph.D. Diss., University of Groningen (1999).

19. Lu, C.W. and Reynolds, M.R.J. "CUSUM charts for monitoring an autocorrelated process", Journal of Quality Technology, 33(3), pp. 316-335 (2001).

20. Lu, C.W. and Reynolds, M.R.J. "EWMA control charts for monitoring the mean of autocorrelated processes", Journal of Quality Technology, 31(2), pp. 166-188 (1999).

21. Areepong, Y. "A comparison of performance of residual control charts for trend stationary AR (p) processes", International Journal of Pure and Applied Mathematics, 85(3), pp. 583-592 (2013).

22. Lin, W.S.W. "The use of combined control charts with forecast-based quality monitoring scheme", Ph.D. diss, University of Alabama (1995).

23. Zhang, N.F. "A statistical control chart for stationary process data", Technometrics, 40(1), pp. 24-38 (1998).

24. Abbas, N., Riaz, M. and Does, R.J.M.M. "Mixed exponentially weighted moving average-cumulative sum charts for process monitoring", Quality and Reliability Engineering International, 29(3), pp. 345-356 (2013).

25. Zaman, B., Riaz, M., Abbas, N. and Does, R.J. "Mixed cumulative sum-exponentially weighted moving average control charts: An efficient way of monitoring process location", Quality and Reliability Engineering International, 31(8), pp. 1407-1421 (2015).

26. Runger, G.C. and Willemain, T.R. "Model-based and model-free control of autocorrelated processes", Journal of Quality Technology, 27(4), pp. 283-292 (1995).

27. Lucas, J.M. "Combined Shewhart-CUSUM quality control schemes", Journal of Quality Technology, 14(2), pp. 51-59 (1982).

28. Lucas, J.M. and Saccucci, M.S. "Exponentially weighted moving average control schemes: Properties and enhancements", Technometrics, 32(1), pp. 1-12 (1990).
29. Wu, Z., Jiao, J.X., Yang, M., Liu, Y. and Wang, Z.J. "An enhanced adaptive CUSUM control chart", IIE Transactions, 41(7), pp. 642-653 (2009).

30. Abbasi, S.A., Riaz, M., Miller, A., Ahmad, S. and Nazir, H.Z. "EWMA dispersion control charts for normal and non-normal processes", Quality and Reliability Engineering International, 31(8), pp. 1691-1704 (2014).

\section{Biographies}

Richard Osei-Aning is currently at the final stages of obtaining MS degree in Applied Statistics from the Department of Mathematics and Statistics at King Fahd University of Petroleum and Minerals, Dhahran, Saudia Arabia. His research interests include time series analysis and statistical process control.

Saddam Akber Abbasi obtained his BS degree with honors in Computer Science from the PMAS-Arid Agriculture University Rawalpindi, Pakistan, in 2005, and his MSc degree in Statistics from Quaid-i-Azam University, Islamabad, Pakistan, in 2007. He obtained his PhD degree in Statistics in the Department of Statistics at the University of Auckland, Auckland, New Zealand, in 2012. He has been serving as an Assistant Professor in the Department of Mathematics and Statistics, King Fahad University of Petroleum and Minerals, Dhahran 31261, Saudi Arabia, since 2013. His research interests include Statistical Process Control (SPC) and artificial neural networks.

Muhammad Riaz received the MSc degree in Statistics from the Department of Mathematics and Statistics at Quaid-i-Azam University, Islamabad, Pakistan, in 2001 and the $\mathrm{PhD}$ in Statistics from the Institute of Business and Industrial Statistics at University of Amsterdam, The Netherlands, in 2008. He served as a Statistical Officer in MINFAL Pakistan during 20022003; as a Staff Demographer in PIDE Pakistan during 2003-2004; as a lecturer in the Department of Statistics, Quaid-i-Azam University, Islamabad, Pakistan during 2004-2007; and as an Assistant Professor in the Department of Statistics, Quaid-i-Azam University, Islamabad, Pakistan during 2007-2010. He has been serving as an Assistant Professor in the Department of Mathematics and Statistics, King Fahad University of Petroleum and Minerals, Dhahran 31261, Saudi Arabia, since 2010. His current research interests include statistical process control, Non-Parametric techniques, experimental designs, and computational statistics with focus on code development. 